\title{
Compressional Alfvén eigenmodes in rotating spherical tokamak plasmas
}

\author{
H. M. Smith ${ }^{1}$, E. D. Fredrickson ${ }^{2}$ \\ 1 Max-Planck/Princeton Center for Plasma Physics, Max-Planck-Institut für \\ Plasmaphysik, 17491 Greifswald, Germany \\ 2 Princeton Plasma Physics Laboratory, Princeton, New Jersey 08543, USA \\ E-mail: hakan.smith@ipp.mpg.de
}

\begin{abstract}
Spherical tokamaks often have a considerable toroidal plasma rotation of several tens of $\mathrm{kHz}$. Compressional Alfvén eigenmodes (CAEs) in such devices therefore experience a frequency shift, which if the plasma were rotating as a rigid body, would be a simple Doppler shift. However, since the rotation frequency depends on minor radius, the eigenmodes are affected in a more complicated way. The eigenmode solver CAE3B [Smith et al. Plasma Phys. Control. Fusion 51, 075001 (2009)] has been extended to account for toroidal plasma rotation. The results show that the eigenfrequency shift due to rotation can be approximated by a rigid body rotation with a frequency computed from a spatial average of the real rotation profile weighted with the eigenmode amplitude. To investigate the effect of extending the computational domain to the vessel wall, a simplified eigenmode equation, yet retaining plasma rotation, is solved by a modified version of the CAE code used in [Fredrickson et al. Phys. Plasmas 20, 042112 (2013)]. In summary, both solving the full eigenmode equation, as in the CAE3B code, and placing the boundary at the vessel wall, as in the CAE code, significantly influences the calculated eigenfrequencies.
\end{abstract}

PACS numbers: 52.35.Bj, 52.35.Hr, 52.55.Fa, 51.10.+y 


\section{Introduction}

Compressional Alfvén eigenmodes (CAEs) are found in the presence of fast ion populations both in conventional tokamaks and in spherical tokamaks. In conventional tokamaks, the fast ions can excite compressional waves close to harmonics of the ion cyclotron frequency $[1,2]$, and the phenomenon is termed ion cyclotron emission (ICE) $[3,4,5,6]$. In spherical tokamaks $[7,8,9,10]$, and also in some conventional tokamak experiments [11], the magnetic field is lower and the fast ion drive causes compressional eigenmodes to appear at frequencies comparable to but below the on-axis ion cyclotron frequency.

The eigenmode structure in large aspect ratio tokamaks is that of a poloidally and toroidally traveling wave with the mode numbers $m$ and $n$, respectively [12]. In a tight-aspect-ratio tokamak, the typical sub-ion cyclotron frequency eigenmodes are also traveling in the toroidal direction, but in the poloidal plane they are localized on the outboard side and have a more standing-wave-like nature [13]. The reason for the localization can be understood from the simple local approximation of the compressional Alfvén wave dispersion relation for frequencies much smaller than the ion cyclotron frequency, $\omega^{2}=-v_{\mathrm{A}}^{2} \nabla^{2}$. Upon splitting the Laplace operator in a toroidal part and a part operating in the poloidal plane, we can write the equation for the perturbed quantity $X$ as

$$
\left(\nabla_{\mathrm{pol}}^{2}-\frac{n^{2}}{R^{2}}+\frac{\omega^{2}}{v_{\mathrm{A}}^{2}}\right) X=0
$$

The quantity $V \equiv n^{2} / R^{2}-\omega^{2} / v_{\mathrm{A}}^{2}$ can be seen as an effective potential for the eigenmode problem expressed in the poloidal plane. The dependence of the Alfvén velocity, $v_{\mathrm{A}}^{2}=B^{2} /\left(\mu_{0} \rho\right)$, on mass density, $\rho$, makes the second term of the potential $V$ zero at the plasma edge, which confines the mode to within the plasma. Combined, the two terms in $V$ have a minimum near the outboard edge of the plasma, with a negative value, and become positive moving inwards and outwards from that point. Consequently, the eigenmodes tend to be localized on the outboard side of the torus.

The localization of CAEs in spherical tokamaks has been studied analytically in $[14,15]$, but these models are by necessity simplified, and comparison with experiments [10] has showed the need for more advanced models. Numerical studies have been able to more accurately include the magnetic-field geometry of the spherical tokamak [16, 13]. However, these numerical studies also employ certain simplifying assumptions. The simulations with the ideal MHD code NOVA [16] neglect the Hall term, which can be of importance since the eigenmode frequency is of the order of the ion cyclotron frequency $\omega_{\text {ci }}$. The CAE3B code (which was presented in [13] but not given a name there), on the other hand, includes the Hall term but excludes shear Alfvén waves by assuming that $v_{\mathrm{A}}^{2} /\left(\omega^{2} B^{2}\right)(\boldsymbol{B} \cdot \nabla)^{2} \ll 1$ when operating on components of the perturbed magnetic field perpendicular to the background field. This restriction is lifted in the WHALES code [17], which is based on finite elements instead of finite differences as in CAE3B. Moreover, nonlinear effects due to the presence of the fast ion population can influence 
the eigenmode structure [18], but such effects are not considered here.

This paper will focus on the effects of plasma rotation on the eigenmodes, taking the National Spherical Tokamak Experiment (NSTX) as an example. The plasmas in NSTX typically revolve toroidally with a frequency of a few tens of $\mathrm{kHz}$, and there are CAEs propagating in the opposite direction to the beam ion injection which have frequencies somewhat below $1 \mathrm{MHz}$ [19]. As a simple approximation, the effect of the rotation is to cause a Doppler shift of the CAE in the lab frame-of-reference with respect to the plasma frame. The frequency shift is the rotation frequency times the toroidal mode number $n$. However, it turns out that this is an oversimplification of the effect that rotation has on CAEs. The plasma does not rotate as a rigid body, but the rotation frequency is a function of plasma radius, and in the present paper it is therefore investigated how this influences the eigenmode frequency and the eigenmode structure. To this end, a new version of the CAE3B code is developed, which includes the effects of the plasma rotation on the eigenmodes. In Section 2 the eigenmode equation derived in [13] is extended to account for plasma rotation, and in Section 3 results from numerical solutions of this equation with the new code are presented and discussed.

One simplification in CAE3B, as well as the other numerical codes mentioned above, is the restriction of the computational domain to within the separatrix. This is a natural limitation of the codes, since they work in magnetic flux coordinates. Solutions of the simplified dispersion relation (1) for a larger region have shown that the eigenmode is likely to extend beyond the separatrix, and that the vessel wall shape may influence the mode structure [19]. To assess the effect of this, the CAE code used in [19] has been modified to solve a simplified version of the CAE3B equations, similar to (1) but including rotation effects. The extension of the computational domain results in a frequency downshift, which is discussed in Section 4.

\section{The eigenmode equation}

Compressional Alfvén eigenmodes are governed by the momentum balance equation, Ohm's law including the Hall term, and Faraday's law

$$
\begin{aligned}
& \rho \frac{\partial}{\partial t} \boldsymbol{v}+\rho \boldsymbol{v} \cdot \nabla \boldsymbol{v}=\boldsymbol{J} \times \boldsymbol{B}-\nabla p, \\
& \boldsymbol{E}=\left(\frac{\boldsymbol{J}}{n_{e} e}-\boldsymbol{v}\right) \times \boldsymbol{B}, \\
& \nabla \times \boldsymbol{E}=-\frac{\partial \boldsymbol{B}}{\partial t} .
\end{aligned}
$$

These equations are linearized by setting $\rho=\rho_{0}+\rho_{1}, \boldsymbol{v}=\boldsymbol{v}_{0}+\boldsymbol{v}_{1}, \boldsymbol{B}=\boldsymbol{B}_{0}+\boldsymbol{B}_{1}$, $\boldsymbol{J}=\boldsymbol{J}_{0}+\boldsymbol{J}_{1}$, and assuming that perturbed quantities vary as $\exp (-i \omega t)$. Only waves that propagate much faster than the ion thermal speed are to be modeled, so the cold plasma approximation is used, i.e. the perturbed pressure is zero, $p=p_{0}$. In [13] it was assumed that $\boldsymbol{v}_{0}=0$, and the linearized equations thus became

$$
-i \omega \rho_{0} \boldsymbol{v}_{1}=\boldsymbol{J}_{1} \times \boldsymbol{B}_{0}+\boldsymbol{J}_{0} \times \boldsymbol{B}_{1}
$$




$$
\begin{aligned}
\boldsymbol{E}_{1} & =\left(\frac{\boldsymbol{J}_{1}}{n_{e} e}-\boldsymbol{v}_{1}\right) \times \boldsymbol{B}_{0}+\frac{\boldsymbol{J}_{0}}{n_{e} e} \times \boldsymbol{B}_{1} . \\
i \omega \boldsymbol{B}_{1} & =\nabla \times \boldsymbol{E}_{1} .
\end{aligned}
$$

One can take $\boldsymbol{v}_{1}$ from (5), insert it into (6) and then insert $\boldsymbol{E}_{1}$ into (7) to obtain the eigenmode equation which is solved in [13],

$\omega^{2} \boldsymbol{B}_{1}=\nabla \times\left\{i F\left[\tilde{\boldsymbol{J}}_{1} \times \boldsymbol{B}_{0}+\tilde{\boldsymbol{J}}_{0} \times \boldsymbol{B}_{1}\right]-G\left[\tilde{\boldsymbol{J}}_{1} \times \boldsymbol{B}_{0}+\tilde{\boldsymbol{J}}_{0} \times \boldsymbol{B}_{1}\right] \times \boldsymbol{B}_{0}\right\}$,

where $\tilde{\boldsymbol{J}}_{0} \equiv \mu_{0} \boldsymbol{J}_{0}, \tilde{\boldsymbol{J}}_{1} \equiv \mu_{0} \boldsymbol{J}_{1}$ and

$$
\begin{aligned}
& F \equiv-\frac{\omega}{\mu_{0} n_{e} e}=-\omega v_{\mathrm{A}}^{2} /\left(\omega_{\mathrm{ci}} B_{0}\right), \\
& G \equiv \frac{1}{\mu_{0} \rho_{0}}=v_{\mathrm{A}}^{2} / B_{0}^{2} .
\end{aligned}
$$

We now include plasma rotation $\boldsymbol{v}_{0}=\Omega(r) R \hat{\varphi}$, where $r$ is a flux surface label with the dimensions of length, $R$ is the major radius coordinate and $\hat{\varphi}$ is a unit vector in the toroidal direction. The coordinate system $(r, \theta, \varphi)$ is chosen to be right-handed with a poloidal coordinate $\theta$ increasing upwards on the outboard side. The linearized momentum balance and Ohm's law become

$$
\begin{aligned}
& \rho_{0}\left(-i \omega \boldsymbol{v}_{1}+\boldsymbol{v}_{0} \cdot \nabla \boldsymbol{v}_{1}+\boldsymbol{v}_{1} \cdot \nabla \boldsymbol{v}_{0}\right)+\rho_{1} \boldsymbol{v}_{0} \cdot \nabla \boldsymbol{v}_{0}=\boldsymbol{J}_{1} \times \boldsymbol{B}_{0}+\boldsymbol{J}_{0} \times \boldsymbol{B}_{1} \\
& \boldsymbol{E}_{1}=\left(\frac{\boldsymbol{J}_{1}}{n_{e} e}-\boldsymbol{v}_{1}\right) \times \boldsymbol{B}_{0}+\left(\frac{\boldsymbol{J}_{0}}{n_{e} e}-\boldsymbol{v}_{0}\right) \times \boldsymbol{B}_{1} .
\end{aligned}
$$

An expansion is now made in the small parameter $\Omega / \omega$, which for CAEs in NSTX is typically not larger than $0.05-0.1$. Consequently, terms of the order $\Omega / \omega$ are kept, but terms of the order $\Omega^{2} / \omega^{2}$ will be neglected. In general, the spatial derivatives are considered to be comparable in a spherical tokamak, $\nabla \sim a^{-1} \sim R^{-1}$, where $a$ is the minor radius. Using these orderings in (11), one sees that the second and third terms in the parenthesis on the left hand side are of order $\Omega / \omega$ compared with the first term and should be kept. In the term $\rho_{1} \boldsymbol{v}_{0} \cdot \nabla \boldsymbol{v}_{0}$, one must use the continuity equation to estimate $\rho_{1} \sim \rho_{0} R^{-1} v_{1} / \omega$. The term $\rho_{1} \boldsymbol{v}_{0} \cdot \nabla \boldsymbol{v}_{0}$ can therefore be neglected in (11), since it is of the order $\Omega^{2} / \omega^{2}$ compared with the first term.

Henceforth, it is assumed that perturbed quantities vary as $\exp (-i n \varphi-i \omega t)$, following the convention in [13]. Thus, $\boldsymbol{v}_{0} \cdot \nabla \boldsymbol{v}_{1}=-i n \Omega \boldsymbol{v}_{1}$ and the form of the rotation velocity $\boldsymbol{v}_{0}=\Omega(r) R \hat{\varphi}$ yields

$$
\nabla \boldsymbol{v}_{0}=\Omega[(\nabla R) \hat{\varphi}-\hat{\varphi} \nabla R]+\Omega^{\prime}(\nabla r) R \hat{\varphi} .
$$

The momentum balance equation becomes

$$
\begin{aligned}
& -i(\omega+n \Omega) \boldsymbol{v}_{1}+\Omega^{\prime} R \boldsymbol{v}_{1} \cdot \nabla r \hat{\varphi}+\Omega \boldsymbol{v}_{1} \cdot[(\nabla R) \hat{\varphi}-\hat{\varphi} \nabla R]= \\
& =G\left[\tilde{\boldsymbol{J}}_{1} \times \boldsymbol{B}_{0}+\tilde{\boldsymbol{J}}_{0} \times \boldsymbol{B}_{1}\right],
\end{aligned}
$$

where the prime denotes radial derivative. Solving this expression for $\boldsymbol{v}_{1}$ (neglecting terms of order $\Omega^{2} / \omega^{2}$ ), we obtain $\boldsymbol{v}_{1}=\overline{\boldsymbol{v}}+\tilde{\boldsymbol{v}}$, where $\overline{\boldsymbol{v}}$ is the perturbed velocity obtained without the $\boldsymbol{v}_{1} \cdot \nabla \boldsymbol{v}_{0}$ term in (11),

$$
\overline{\boldsymbol{v}}=\frac{G\left[\tilde{\boldsymbol{J}}_{1} \times \boldsymbol{B}_{0}+\tilde{\boldsymbol{J}}_{0} \times \boldsymbol{B}_{1}\right]}{-i(\omega+n \Omega)}
$$


and

$$
\tilde{\boldsymbol{v}}=\frac{-\hat{\varphi}\left(\Omega^{\prime} R \nabla r+\Omega \nabla R\right)+\Omega \nabla R \hat{\varphi}}{-i(\omega+n \Omega)} \cdot \overline{\boldsymbol{v}} .
$$

When $\boldsymbol{E}_{1}$ from (12) is inserted into Faraday's law to obtain the eigenmode equation, there are two conceptually new terms compared with the derivation of $(8),-\nabla \times\left(\boldsymbol{v}_{0} \times\right.$ $\left.\boldsymbol{B}_{1}\right)$ and $-\nabla \times\left(\tilde{\boldsymbol{v}} \times \boldsymbol{B}_{0}\right)$. The former is

$$
\begin{aligned}
& -\nabla \times\left(\boldsymbol{v}_{0} \times \boldsymbol{B}_{1}\right)=-\boldsymbol{B}_{1} \cdot \nabla \boldsymbol{v}_{0}+\boldsymbol{v}_{0} \cdot \nabla \boldsymbol{B}_{1}= \\
& =-\boldsymbol{B}_{1} \cdot\left(\Omega^{\prime} R \nabla r+\Omega \nabla R\right) \hat{\varphi}+\Omega \boldsymbol{B}_{1} \cdot \hat{\varphi} \nabla R-i n \Omega \boldsymbol{B}_{1},
\end{aligned}
$$

and it can be shown that for $-\nabla \times\left(\tilde{\boldsymbol{v}} \times \boldsymbol{B}_{0}\right)$ the following holds,

$$
\begin{aligned}
& -i(\omega+n \Omega)\left(-\nabla \times\left(\tilde{\boldsymbol{v}} \times \boldsymbol{B}_{0}\right)\right)= \\
& \nabla r \times \nabla\left[\psi^{\prime}\left(\Omega^{\prime}+\nu \Omega\right) \nabla r \cdot \overline{\boldsymbol{v}}+\mu \Omega \boldsymbol{B}_{0} \cdot \overline{\boldsymbol{v}}\right]+ \\
& +\nabla \times\left[\boldsymbol{\Lambda} \Omega B_{0}^{-2} \overline{\boldsymbol{v}} \cdot\left(I \boldsymbol{B}_{0}-\psi^{\prime} \boldsymbol{\Lambda}\right)\right] .
\end{aligned}
$$

In the above expression, the following notation was introduced for brevity

$$
\begin{aligned}
\nu & \equiv \nabla r \cdot \nabla R /\left(R|\nabla r|^{2}\right), \\
\mu & \equiv \nabla r \times \nabla R \cdot \hat{\varphi} /|\nabla r|^{2}, \\
\boldsymbol{\Lambda} & \equiv \boldsymbol{B}_{0} \times \nabla r,
\end{aligned}
$$

and it was used that the equilibrium magnetic field has the form $\boldsymbol{B}_{0}=I(r) \nabla \varphi+$ $\psi^{\prime}(r) \nabla \varphi \times \nabla r$, which leads to the expression for the toroidal unit vector $\hat{\varphi}=\left(I(r) \boldsymbol{B}_{0}-\right.$ $\left.\psi^{\prime} \boldsymbol{\Lambda}\right) /\left(R B_{0}^{2}\right)$.

Faraday's law becomes

$$
\begin{aligned}
& i \omega \boldsymbol{B}_{1}=\frac{1}{-i \omega} \nabla \times\left\{i F\left[\tilde{\boldsymbol{J}}_{1} \times \boldsymbol{B}_{0}+\tilde{\boldsymbol{J}}_{0} \times \boldsymbol{B}_{1}\right]-K G\left[\tilde{\boldsymbol{J}}_{1} \times \boldsymbol{B}_{0}+\tilde{\boldsymbol{J}}_{0} \times \boldsymbol{B}_{1}\right] \times \boldsymbol{B}_{0}\right\}+ \\
& +\frac{K}{-i \omega} \nabla r \times \nabla\left[\psi^{\prime}\left(\Omega^{\prime}+\nu \Omega\right) \nabla r \cdot \overline{\boldsymbol{v}}+\mu \Omega \boldsymbol{B}_{0} \cdot \overline{\boldsymbol{v}}\right]+ \\
& +\frac{K}{-i \omega} \nabla \times\left[\boldsymbol{\Lambda} \Omega B_{0}^{-2} \overline{\boldsymbol{v}} \cdot\left(I \boldsymbol{B}_{0}-\psi^{\prime} \boldsymbol{\Lambda}\right)\right]+ \\
& -\boldsymbol{B}_{1} \cdot\left(\Omega^{\prime} R \nabla r+\Omega \nabla R\right) \hat{\varphi}+\Omega \boldsymbol{B}_{1} \cdot \hat{\varphi} \nabla R-i n \Omega \boldsymbol{B}_{1},
\end{aligned}
$$

where $K(\omega)=\omega /(\omega+n \Omega)$. To obtain a form where the eigenvalue $\omega^{2}$ appears on the left hand side, the last term is moved to the left and the equation is multiplied by $-i \omega K$. Finally, the eigenmode equation becomes

$$
\begin{aligned}
& \omega^{2} \boldsymbol{B}_{1}=K \nabla \times\left\{i F\left[\tilde{\boldsymbol{J}}_{1} \times \boldsymbol{B}_{0}+\tilde{\boldsymbol{J}}_{0} \times \boldsymbol{B}_{1}\right]\right\}+ \\
& -K^{2} \nabla \times\left\{G\left[\tilde{\boldsymbol{J}}_{1} \times \boldsymbol{B}_{0}+\tilde{\boldsymbol{J}}_{0} \times \boldsymbol{B}_{1}\right] \times \boldsymbol{B}_{0}\right\}+ \\
& -K^{3} \frac{n \Omega^{\prime}}{\omega} \boldsymbol{B}_{0} G\left[\tilde{\boldsymbol{J}}_{1} \times \boldsymbol{B}_{0}+\tilde{\boldsymbol{J}}_{0} \times \boldsymbol{B}_{1}\right] \cdot \nabla r+ \\
& +K^{2} \nabla r \times \nabla\left[\psi^{\prime}\left(\Omega^{\prime}+\nu \Omega\right) \nabla r \cdot \overline{\boldsymbol{v}}+\mu \Omega \boldsymbol{B}_{0} \cdot \overline{\boldsymbol{v}}\right]+ \\
& +K^{2} \nabla \times\left[\boldsymbol{\Lambda} \Omega B_{0}^{-2} \overline{\boldsymbol{v}} \cdot\left(I \boldsymbol{B}_{0}-\psi^{\prime} \boldsymbol{\Lambda}\right)\right]+ \\
& +i \omega K \boldsymbol{B}_{1} \cdot\left[\left(\Omega^{\prime} R \nabla r+\Omega \nabla R\right) \hat{\varphi}-\Omega \hat{\varphi} \nabla R\right] .
\end{aligned}
$$


One recognizes that for rigid body rotation with a small rotation frequency $\Omega$, only the two first lines remain, and the solutions are the same as to (8), except that the frequency is shifted by an amount $-n \Omega$. The eigenmode equation (21) has been split into its three vector components in Appendix A, and the resulting equations for the three components of the perturbed magnetic field have been implemented in the CAE3B code. These magnetic field components are represented by the quantities $b_{r}, b_{\wedge}$ and $b_{\|}$, defined by

$$
\boldsymbol{B}_{1}=b_{r} \nabla r+b_{\wedge} \boldsymbol{\Lambda}+b_{\|} \boldsymbol{B}_{0} .
$$

However, for the presentation of the results in the next Section, the corresponding quantities in physical units $B_{r}=b_{r}|\nabla r|, B_{\wedge}=b_{\wedge}|\nabla r| B_{0}$ and $B_{\|}=b_{\|} B_{0}$ will be used. The boundary condition $B_{\|}=0$, which was used in [13], is also employed in the following calculations.

\section{Results from CAE3B with rotation}

To illustrate the effects of plasma rotation, an NSTX equilibrium from discharge 130335 at $t=480 \mathrm{~ms}$ is used, with $\boldsymbol{B} \cdot \hat{\varphi}>0, \boldsymbol{J} \cdot \hat{\varphi}<0$ and $f_{\text {rot }}=\Omega /(2 \pi)<0$. In the experiment, there were CAEs with frequencies in the range $800 \mathrm{kHz}$ to $1100 \mathrm{kHz}$ and with toroidal mode numbers $n=-3$ to -5 (following the definition that perturbed quantities vary as $\exp (-i n \varphi-i \omega t)$ with $\omega>0)$. Consequently, these eigenmodes travel in the direction of increasing $\varphi$ and are thus counter-beam-propagating. The plasma, on the other hand, rotates in the direction of decreasing $\varphi$. The ion cyclotron frequency varies in this equilibrium from $2.5 \mathrm{MHz}$ on the outboard side to $9 \mathrm{MHz}$ on the inboard side. The experimental profiles of rotation frequency and density are presented in figure 1 as a function of major radius on the mid-plane. Measurements of the rotation profile (dashed in figure 1a) are only available in the outer midplane. The profile used in the CAE3B calculations (solid) has therefore been extrapolated to the inboard side by requiring it to be a flux function, and it was also adjusted to be zero at the boundary. In figure 1a, there is also an alternative rotation profile (dash-dotted), which is used later for a comparison in figure 5. The magnitude of the three vector components of $\boldsymbol{B}_{1}$ for a typical solution for $n=-4$ is presented in figure 2. Note that the parallel field is much larger than the perpendicular components.

A few examples of solutions for $n=-4$ are shown in figure 3 , which displays the magnitude of the parallel component of the perturbed magnetic field (the eigenmode in figure 2 is seen again in figure 3c). According to the naming convention of [13], the five eigenmodes have the mode numbers $(s=1 / 2, m=0),(s=1, m=1),(s=1, m=-1)$, $(s=1, m=2)$ and $(s=1, m=-2)$, respectively. However, we will for simplicity just number the eigenmodes in ascending order according to their eigenfrequencies, with the aid of the symbols seen in the figure. The five eigenmode types in figure 3 are indicated with $\circ,+, \triangleright, \times, \square$.

Figure 4 illustrates how the different eigenmode frequencies are affected by the magnitude of the rotation frequency. The calculations were performed for a range 

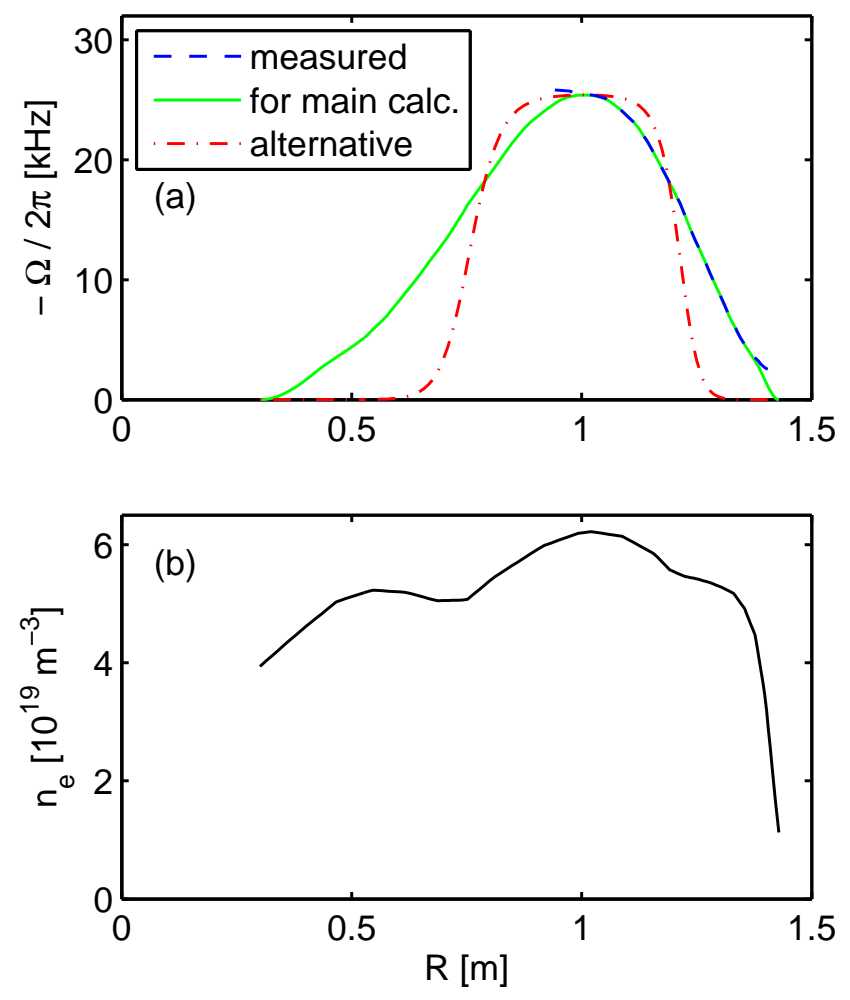

Figure 1. Radial profiles shown as a function of major radius on the mid-plane. (a) Measured rotation frequency on the outboard side (dashed), the corresponding flux function for the rotation frequency used in most CAE3B calculations (solid) and an alternative rotation profile used only in the comparison in figure 5 (dash-dotted). (b) Density profile.
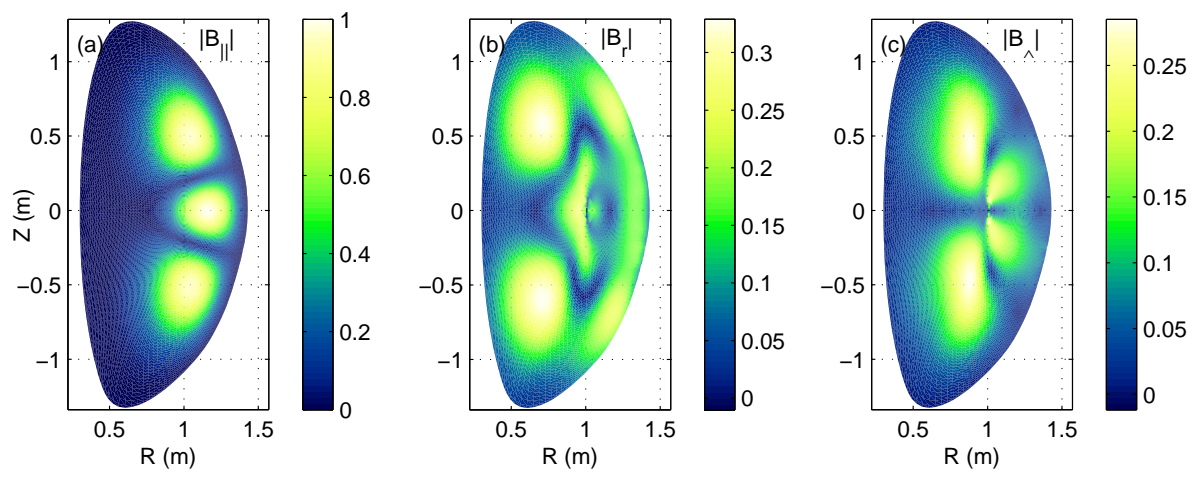

Figure 2. Magnitude of the three components of the perturbed magnetic field (a) $\left|B_{\|}\right|$, (b) $\left|B_{r}\right|$, (c) $\left|B_{\wedge}\right|$ for an eigenmode solution for $n=-4$ with $f=1026 \mathrm{kHz}$, also shown in figure $3(\mathrm{c})$. Note the different color scales. 

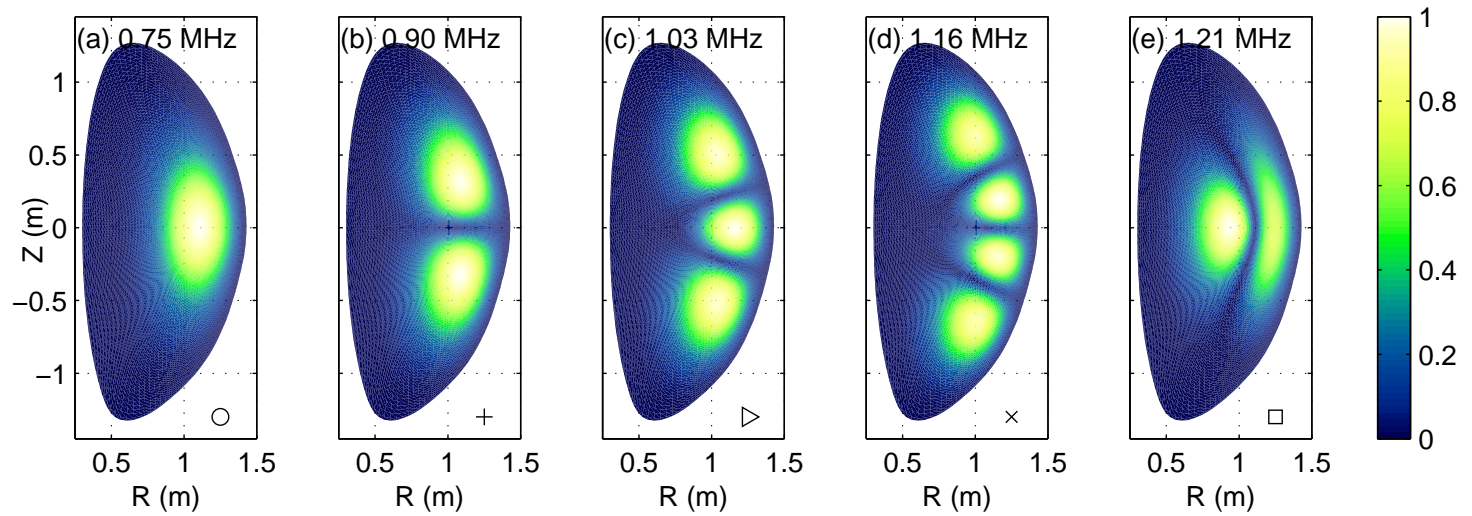

Figure 3. $\left|B_{\|}\right|$for the eigenmode structures with the lowest frequencies for $n=-4$.

of rotation frequencies at the magnetic axis, from the experimental value $f_{\text {rot,axis }}=$ $-25.4 \mathrm{kHz}$ to the oppositely directed $f_{\text {rot,axis }}=25.4 \mathrm{kHz}$. The radial profile was linearly scaled, i.e., the relative shape of the rotation profile stayed the same as in figure 1 . The eigenfrequencies corresponding to the five types of modes (indicated with the different symbols from figure 3) are shown in figure 4 for $n=-3$ to -5 . The different toroidal mode numbers are represented with different line colors.

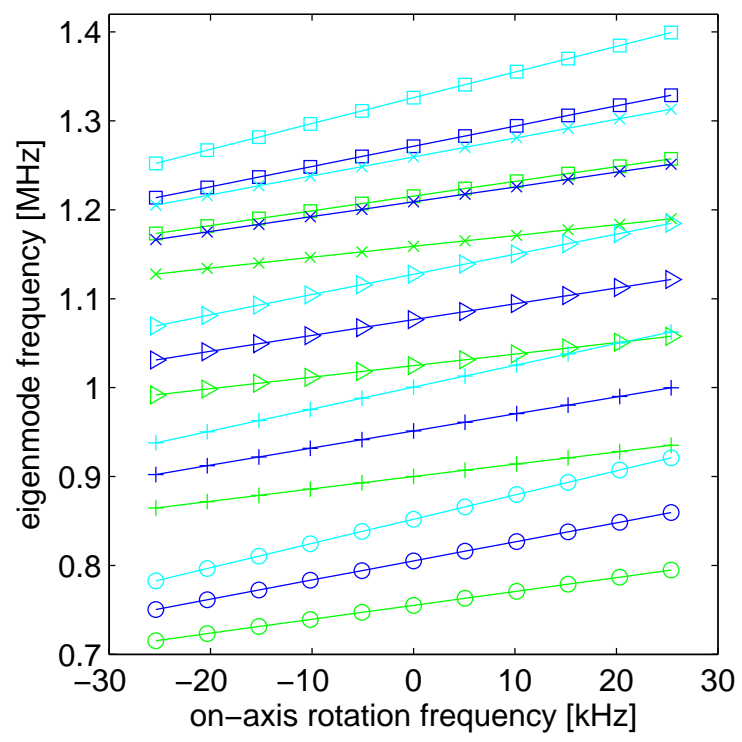

Figure 4. Eigenmode frequencies versus the magnitude of the on-axis rotation frequency for toroidal mode numbers: $n=-3$ (green), -4 (blue), -5 (cyan). The five eigenmode types (columns) in figure 3 are indicated with $\circ,+, \triangleright, \times$ and $\square$, respectively.

It is clear from figure 4 that the eigenfrequency is raised by an increased plasma rotation in the same direction as the eigenmode rotation. The experimental rotation is in the opposite direction to the eigenmode rotation and the eigenfrequencies are therefore lower than they would be without plasma rotation. For $n=-4$, in figures $3 \mathrm{a}-$ 
e, the eigenfrequency is shifted by $\Delta f \simeq-40$ to $-55 \mathrm{kHz}$ with respect to the nonrotating case. We can define an effective rotation frequency that the mode experiences, $f_{\text {rot,eff }}=-\Delta f / n$, which is the rigid body rotation that would yield the same frequency shift $\Delta f$. For the eigenmodes in figure 3 we obtain for the case of the experimental rotation frequency $f_{\text {rot,axis }}=-25.4 \mathrm{kHz}$ a value $f_{\text {rot,eff }} \simeq-10$ to $-14 \mathrm{kHz}$ (yielding $f_{\text {rot,eff }} / f_{\text {rot,axis }} \simeq 0.4-0.5$ ). One can obtain an approximation for the effective rotation frequency by taking a spatial average of the rotation profile weighted with the $\left|B_{\|}\right|$ amplitude of the eigenmode. Assuming that the plasma rotates with this approximate effective frequency as a rigid body, one can then calculate the corresponding Doppler shift away from the solution at zero rotation. This gives for $n=-4$ the dashed magenta curve in figure 5. It agrees approximately with the blue markers without lines, which are the CAE3B results calculated without the terms $\boldsymbol{v}_{1} \cdot \nabla \boldsymbol{v}_{0}$ in (11) and $\boldsymbol{B}_{1} \cdot \nabla \boldsymbol{v}_{0}$ in (17), i.e. without effects such as rotation shear. The main difference between the solutions to the eigenmode equation (8) without rotation and the version including rotation, equation (21), originates from the first two lines of the latter equation. This seems to be captured roughly by the above described weighted average rotation approximation. But the remaining part of (21), related to the $\boldsymbol{v}_{1} \cdot \nabla \boldsymbol{v}_{0}$ and $\boldsymbol{B}_{1} \cdot \nabla \boldsymbol{v}_{0}$ contributions, results in a correction to the eigenfrequency (the full CAE3B solution shown as blue lines in figure 5). For the experimental rotation frequency $f_{\text {rot,axis }}=-25.4 \mathrm{kHz}$, there is a shift of $\sim 2 \mathrm{kHz}$ in the effective rotation frequency when the smaller terms of the equation are turned off.

Because of these small correction terms, we conclude that it is non-trivial to determine the value of $f_{\text {rot,eff }}$ without performing the full eigenmode calculation. Nevertheless, a qualitative statement can be made that the modes which are more centrally localized tend to have a larger $f_{\text {rot,eff }}$, i.e. a larger slope in figures 4 and 5 . In figure 4 , compare the $n=-4, \times$-marked line (corresponding to figure $3 \mathrm{~d}$ ) and the $n=-4, \square$-marked line (corresponding to figure $3 \mathrm{e}$ ).

To examine the dependence on the rotation profile further, an alternative profile was introduced in figure 1, which has the rotation more concentrated to the central plasma. A comparison with results for this profile is made for $n=-4$ in figure 5 . The eigenmode frequencies in figure 5 for the new rotation profile (red solid lines) have a value of $f_{\text {rot,eff }} / f_{\text {rot,axis }}$ which is around two thirds of the value for the solutions for the experimental rotation profile (blue solid lines). This is to be expected since the eigenmodes are localized towards the edge where the rotation is lower for the alternative profile.

The frequency is expected to differ between eigenmodes with positive and negative toroidal mode numbers because of the Hall term [13]. However, figure 5 shows that for example for the eigenfrequencies for $n=+4$ (black) and for $n=-4$ (blue solid) this difference is small compared to the effect of plasma rotation. For $n=+4$, the relative sign of plasma rotation and eigenmode rotation is the opposite, and the slope is thus the opposite compared with the $n=-4$ case. The effective rotation frequency for the $n>0$ eigenmodes is very similar to the one for the negative toroidal mode numbers. 


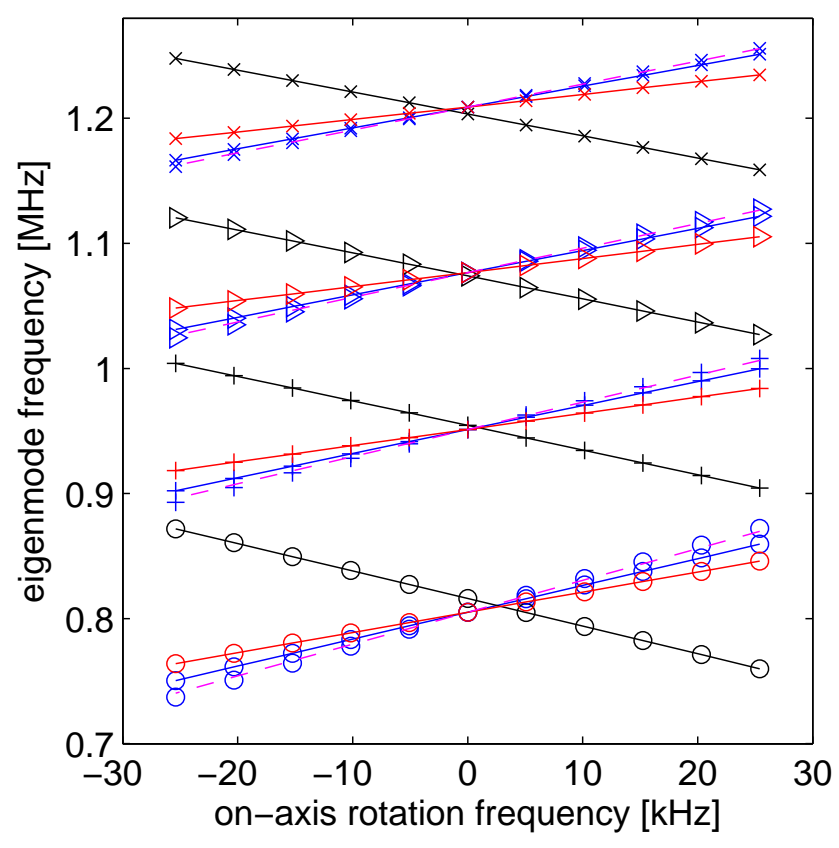

Figure 5. CAE3B eigenfrequencies for $n=+4$ (black) and for $n=-4$ (blue solid). Solutions are also shown for $n=-4$ without the $\boldsymbol{B}_{1} \cdot \nabla \boldsymbol{v}_{0}$ and $\boldsymbol{v}_{1} \cdot \nabla \boldsymbol{v}_{0}$ terms in the eigenmode equation (blue markers, no line). Results obtained using the weighted average rotation Doppler shift approximation described in the text are shown as magenta dashed lines without marker symbols. Note the approximate agreement between the magenta dashed lines and the blue markers without lines. Results for the alternative rotation profile in figure 1 are shown as red lines. As before, the first four eigenmode types in figure 3 are indicated with $\circ,+, \triangleright$ and $\times$.

The effective rotation frequency is also not affected by turning on or off the Hall term (or the terms related to $\boldsymbol{J}_{0}$ ) in the solver. This only gives an overall shift of the frequencies on the order of $\sim 10-20 \mathrm{kHz}$, which can be seen as the difference between the $n= \pm 4$ curves at zero rotation in figure 5 .

That different eigenmode types with the same $n$ can have different effective rotation frequencies (see the different slopes in figure 4) implies that the eigenfrequencies of different modes can approach each other at certain rotation frequencies. Figure 6 shows an example for $n=-4$, where the $\times$ and $\square$ mode frequencies are close to each other at rotation frequencies around $-114 \mathrm{kHz}$ (4.5 times the experimental one). In going from left to right in figure 6 the corresponding mode structures, shown in figure 7 , appear to exchange identities.

One can understand it in the following way. Given the higher $f_{\text {rot,eff }} / f_{\text {rot,axis }}$ slope of the $\square$ mode at slow rotation, one would expect its $f\left(f_{\text {rot,axis }}\right)$ curve to cross the corresponding curve for the $\times$ mode at around $-114 \mathrm{kHz}$. However, such a degeneracy is not allowed, as figure 6 shows. Instead, the curves avoid crossing and the visual appearance of $\left|B_{\|}\right|$for the $(\times)$mode continuously changes its character when going from figure $7 \mathrm{a}$ (at $\left.f_{\text {rot,axis }}=-124 \mathrm{kHz}\right)$ to the way it looks in figure $7 \mathrm{e}$ (at $\left.f_{\text {rot,axis }}=-104 \mathrm{kHz}\right)$. One can make an analogy here to the gaps opening up in the shear Alfvén continuum at 


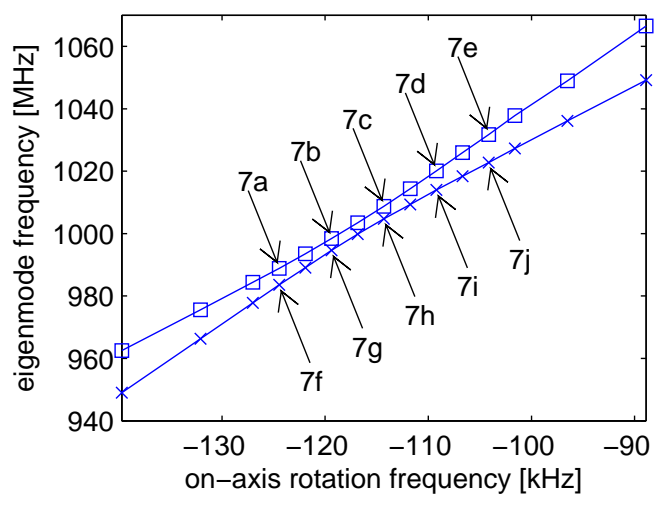

Figure 6. Eigenmode frequencies versus the magnitude of the on-axis rotation frequency for $n=-4$, showing that the eigenfrequencies for the $\times$ and $\square$ modes do not cross.
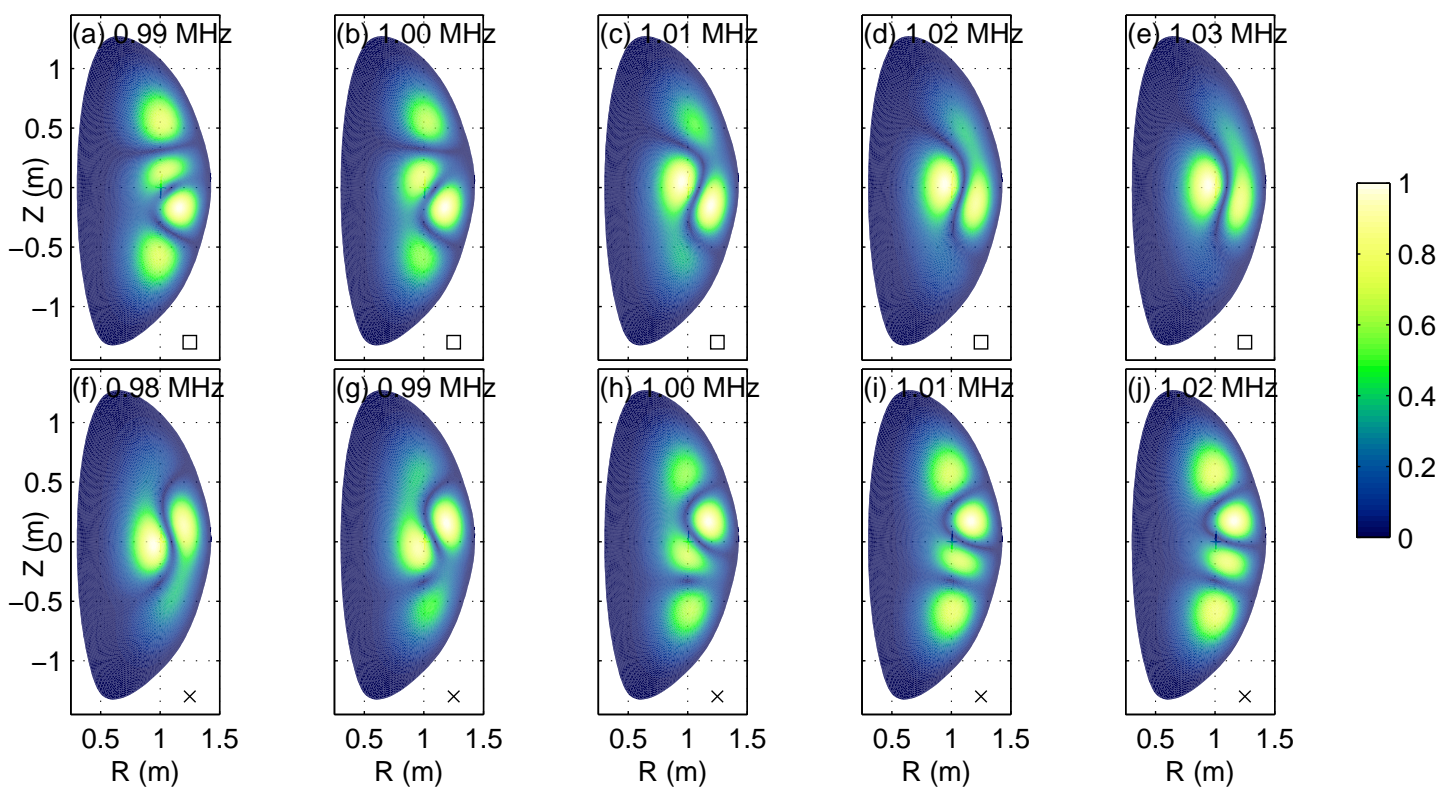

Figure 7. $\left|B_{\|}\right|$for eigenmode structures $(n=-4)$ which transition from one form to another as the rotation frequency is varied. Rotation frequencies from left to right are equally spaced from $-124 \mathrm{kHz}$ to $-104 \mathrm{kHz}$, see also figure 6 . The transition modes resemble superpositions of the two simpler mode structures in figure $3 \mathrm{~d}$ and e.

radii where the toroidicity couples different poloidal mode numbers. In a similar way for the CAEs studied here, geometry effects resolve potential degeneracies between modes, and in the transition region where the eigenfrequencies are close, the modes structures appear to be superpositions of the two involved modes.

\section{The boundary at the wall}

The placement of the boundary within the last closed flux surface instead of at the vessel wall does of course affect the eigenmode solutions. To investigate this, the code 
used in [19], called the CAE code, has been modified to solve a simplified version of the CAE3B equations (21). It does not include the terms stemming from $\boldsymbol{v}_{1} \cdot \nabla \boldsymbol{v}_{0}$ in (11) and $\boldsymbol{B}_{1} \cdot \nabla \boldsymbol{v}_{0}$ in (17), and the effective rotation frequency can thus be expected to be higher (see figure 5). The Hall term and the terms related to $\boldsymbol{J}_{0}$ are also neglected, so that the equation becomes

$$
(\omega+n \Omega)^{2} \boldsymbol{B}_{1}=\nabla \times\left[G\left(\nabla \times \boldsymbol{B}_{1}\right)_{\perp}\right] .
$$

The three components of this equation are (c.f. equations (10)-(12) in [13])

$$
\begin{aligned}
& (\omega+n \Omega)^{2} g^{r r} b_{r}=\left(\boldsymbol{B}_{0} \cdot \nabla\right) v_{\mathrm{A}}^{2} \nabla r \cdot \nabla b_{\|} \\
& (\omega+n \Omega)^{2} g^{r r} b_{\wedge}=B_{0}^{-2}\left(\boldsymbol{B}_{0} \cdot \nabla\right) v_{\mathrm{A}}^{2}\left(\boldsymbol{B}_{0} \times \nabla r\right) \cdot \nabla b_{\|} \\
& (\omega+n \Omega)^{2} B_{0}^{2} b_{\|}=\nabla \cdot\left\{v_{\mathrm{A}}^{2}\left[-B_{0}^{2} \nabla_{\perp} b_{\|}+\nabla r\left(\boldsymbol{B}_{0} \cdot \nabla\right) b_{r}+\left(\boldsymbol{B}_{0} \times \nabla r\right)\left(\boldsymbol{B}_{0} \cdot \nabla\right) b_{\wedge}\right]\right\},
\end{aligned}
$$

where $g^{r r} \equiv|\nabla r|^{2}$. The CAE code in [19], which encompasses the space all the way out to the wall, solves one equation for one unknown, so the above set of three equations in three unknowns needs to be reduced. We have to estimate the influence of the transversal magnetic field components $b_{r}$ and $b_{\wedge}$ on the parallel component $b_{\|}$in (26) to obtain one equation for $b_{\|}$. As a crude approximation, one can treat the eigenmodes as plane waves, but one must keep the spatial variation of $v_{\mathrm{A}}^{2} B_{0}^{2}$ in (26), because it is what shapes the potential well. We thus allow ourselves to replace $\left(\boldsymbol{B}_{0} \cdot \nabla\right)$ with $i k_{\|} B_{0}$ in order to rewrite (24) and (25) as

$$
\begin{aligned}
& (\omega+n \Omega)^{2} g^{r r} b_{r}=i k_{\|} v_{\mathrm{A}}^{2} B_{0} \nabla r \cdot \nabla b_{\|} \\
& (\omega+n \Omega)^{2} g^{r r} b_{\wedge}=B_{0}^{-2} i k_{\|} v_{\mathrm{A}}^{2} B_{0}\left(\boldsymbol{B}_{0} \times \nabla r\right) \cdot \nabla b_{\|},
\end{aligned}
$$

and (26) consequently as

$$
\begin{aligned}
& (\omega+n \Omega)^{2} B_{0}^{2} b_{\|}= \\
= & \nabla \cdot\left\{v _ { \mathrm { A } } ^ { 2 } \left[-B_{0}^{2} \nabla_{\perp} b_{\|}+\right.\right. \\
& \left.\left.-\frac{v_{\mathrm{A}}^{2} B_{0}^{2} k_{\|}^{2}}{(\omega+n \Omega)^{2} g^{r r}}\left(\nabla r \nabla r \cdot \nabla b_{\|}+B_{0}^{-2}\left(\boldsymbol{B}_{0} \times \nabla r\right)\left(\boldsymbol{B}_{0} \times \nabla r\right) \cdot \nabla b_{\|}\right)\right]\right\}= \\
= & -\nabla \cdot\left\{v_{\mathrm{A}}^{2} B_{0}^{2}\left(1+\frac{v_{\mathrm{A}}^{2} k_{\|}^{2}}{(\omega+n \Omega)^{2}}\right) \nabla_{\perp} b_{\|}\right\} .
\end{aligned}
$$

One can estimate that if $(\omega+n \Omega)^{2} \approx k_{\perp}^{2} v_{\mathrm{A}}^{2}$ then, roughly,

$$
\nabla \cdot\left(1+\frac{v_{\mathrm{A}}^{2} k_{\|}^{2}}{(\omega+n \Omega)^{2}}\right) \nabla_{\perp} \approx-k^{2} \approx \nabla^{2},
$$

and it can thus be expected that the inclusion of the perpendicular terms of the perturbed magnetic field into the equation for $b_{\|}$approximately modifies it to become

$$
(\omega+n \Omega)^{2} B_{0}^{2} b_{\|}=-\nabla \cdot v_{\mathrm{A}}^{2} B_{0}^{2} \nabla b_{\|} .
$$

The solutions to this equation with the CAE code are benchmarked in figure 8 against a simplified version of CAE3B, which solves the same equation. The benchmark calculations are performed with the boundary at the plasma edge. Because the 

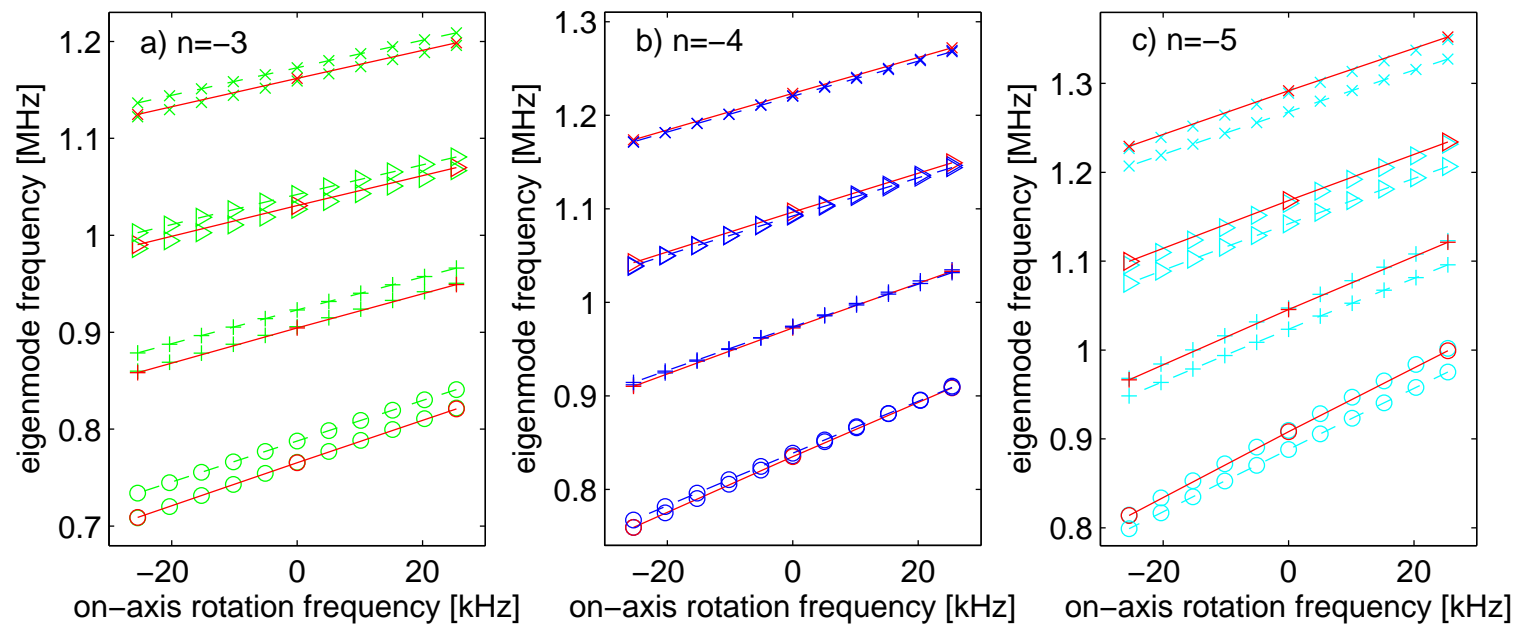

Figure 8. Code and model comparisons with the boundary at the plasma edge. The CAE3B solution to the model equations (24-25) (dashed), the CAE3B solution to the simplified model equation (31) (no line), and a benchmark to the CAE code solution to (31) (red solid). The line colors of the CAE3B solutions correspond to the different toroidal mode numbers, as presented in figure 4. The agreement between the CAE code solution and the CAE3B solution to the simplified model equation (31) is good for all three toroidal mode numbers.

derivation of (31) is heuristic rather than rigorous, a comparison is also made in figure 8 with the CAE3B solution to (24)-(25). One can conclude that the effective rotation frequency and the frequency splitting between different mode structures from the simplified model equation (31) agree rather well with the results of the more complete model in (24)-(25), but the simplified model tends to overestimate the frequency splitting between different $n$.

We are now in the position to compare eigenmode solutions to (31) with the boundary at the wall and at the plasma edge using the CAE code. The quantity $v_{\mathrm{A}}^{2} B_{0}^{2}$ in (31) is proportional to $1 / \rho$, and in the case when the boundary is at the wall, $\rho$ is taken to fall of on a $5 \mathrm{~cm}$ length scale outside the separatrix. At the wall, one would ideally want to set the tangential electric field to zero. However, this is not possible without retaining all three components of $\boldsymbol{B}_{1}$. For simplicity the boundary condition is therefore taken to be $B_{\|}=0$, as in the case with the boundary at the plasma edge.

Figure 9 compares the CAE code eigenmode frequencies (solutions of (31)) with the boundary at the wall and at the plasma edge. Also shown are the full CAE3B (with boundary at the plasma edge) solution from figure 4. Firstly, when comparing the full and simplified model solutions, we see that the main difference lies in the effective rotation frequency (the slope of the curves). To capture the effect of rotation one thus needs to solve the full eigenmode equations, since the simplified model leads to an

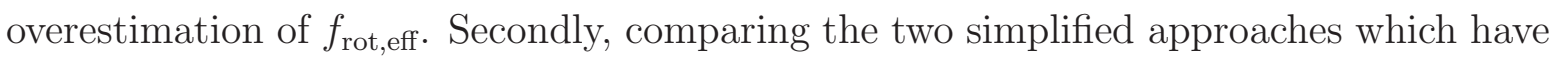
different boundaries, we find that the extension of the domain to the wall lowers the frequencies by $\sim 10-20 \mathrm{kHz}$. The effective rotation frequency, however, is not much affected by the position of the boundary (the slopes of the solid and dashed lines are 
very similar in figure 9).
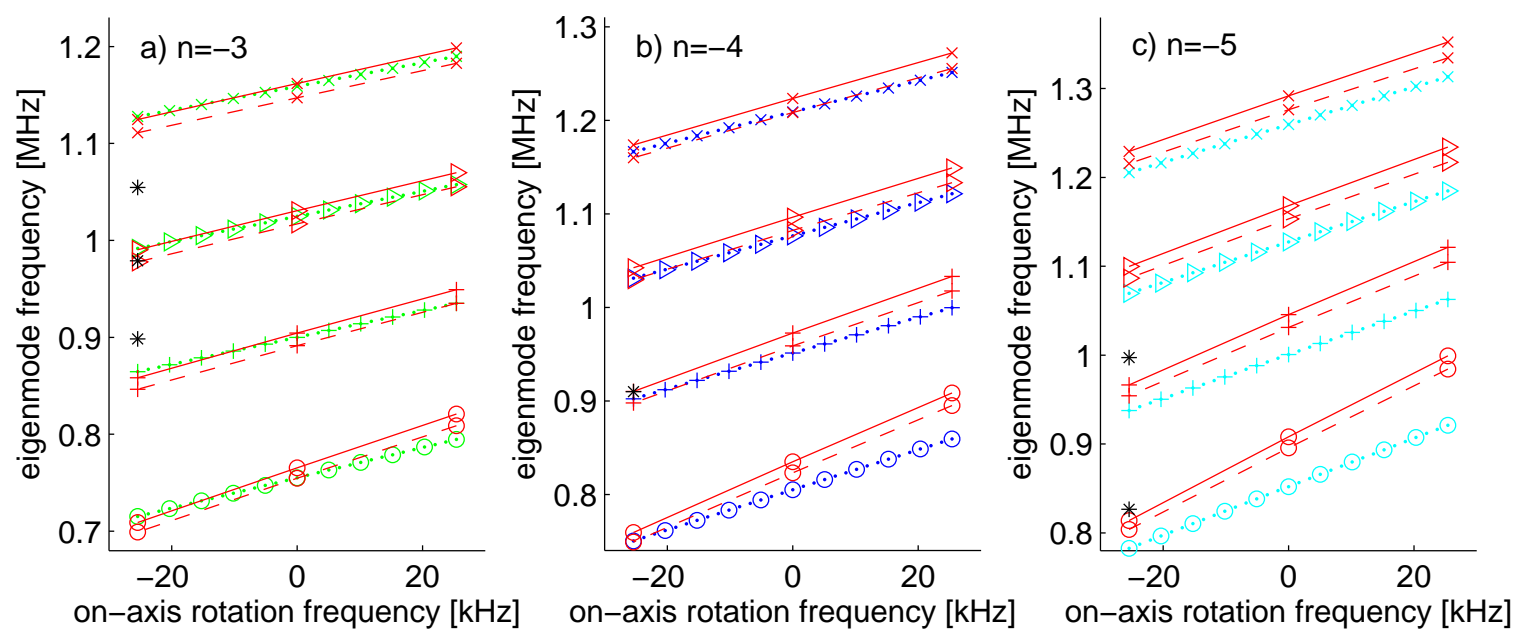

Figure 9. Eigenmode frequencies versus the magnitude of the on-axis rotation frequency. The full CAE3B solution (dotted) has the boundary at the plasma edge. (The line colors of the CAE3B solutions correspond to the different toroidal mode numbers, as presented in figure 4.) The solution to (31) with the CAE code from [19] are shown for the boundary at the wall (dashed) and with the boundary at the plasma edge (solid). The stars show experimental data.

The experimentally measured frequencies for the studied NSTX discharge are presented in a spectrogram in figure 10. Mirnov coil data were analyzed as described in [20] to obtain the toroidal mode numbers, which are indicated in the spectrogram with different colors. For the studied time $t=0.48 \mathrm{~s}$, the frequencies have been transferred to figure 9 and marked as stars. There are fewer modes detected experimentally than one can find with the eigenmode analysis, because the eigenmodes have to be excited to be seen in the experiment, and whether this happens or not depends on details of the fast particle distribution. For $n=-4$ and $n=-5$, the calculations seem to be in rough agreement with the measurements, but for $n=-3$, neither the the eigenfrequencies computed with the full CAE3B code (dotted lines) nor the CAE calculation with the boundary at the wall (dashed lines) match the experimental frequencies. The frequency splitting between the calculated eigenmodes is in this case larger than in the measurements. More systematic comparisons between experiments and numerical results are needed, but such a study is out of the scope of the present work.

Future modeling efforts will need to solve the full eigenmode equation including plasma rotation, since this influences the effective rotation frequency, and such modeling should also place the domain boundary at the wall, since this lowers the eigenmode frequency. Neither of the codes used in this work is able to perform both these tasks at the same time. Furthermore, it would be preferable if the $v_{\mathrm{A}}^{2} /\left(\omega^{2} B^{2}\right)(\boldsymbol{B} \cdot \nabla)^{2} \ll 1$ approximation, which prevents shear waves, could be removed, as is done in the WHALES code [17]. 


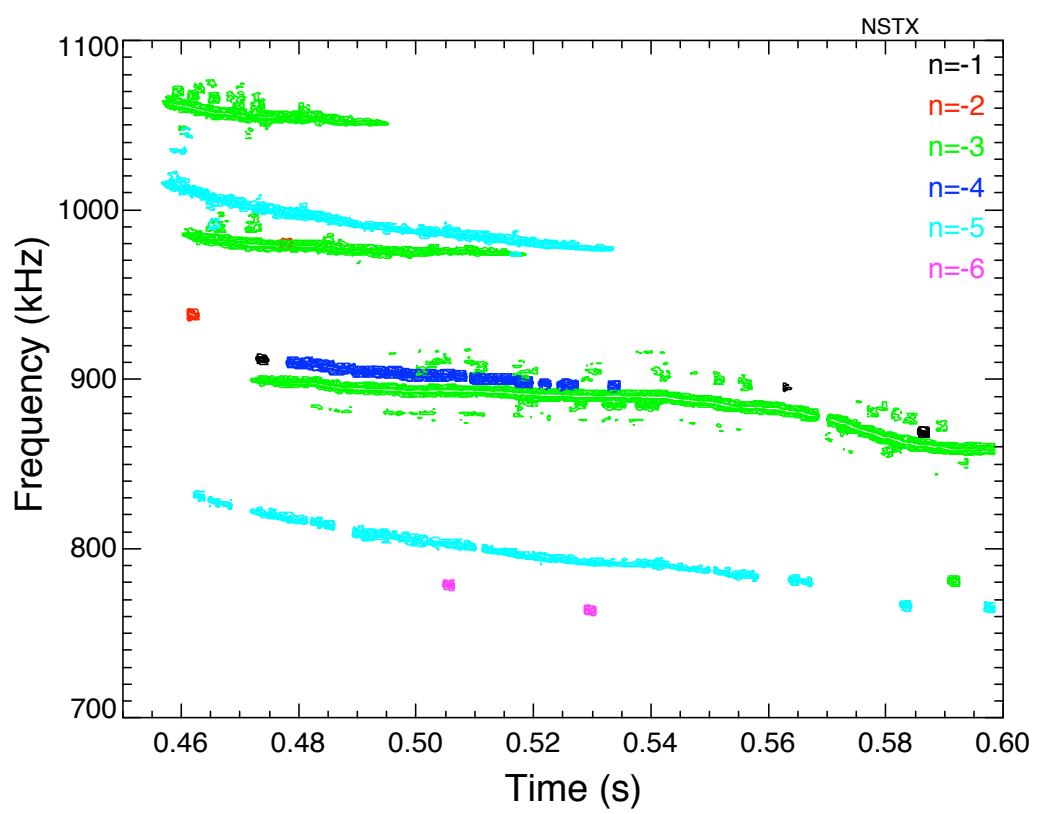

Figure 10. Experimentally measured CAE spectrum, analyzed to display the toroidal mode number, $n=-3$ (green), -4 (blue), -5 (cyan). These colors were used also in previous figures for CAE3B solutions.

\section{Conclusions}

The eigenmodes calculated with CAE3B using the experimental plasma rotation profile are structurally very similar to the ones obtained without taking plasma rotation into account, but their eigenfrequencies differ. However, eigenmodes that are close in frequency can interchange their structural appearance when the rotation frequency is increased. This is for instance the case for the fourth and fifth eigenmode types (denoted with $\times$ and $\square$ in the figures), which swap identities at around 4.5 times the experimental rotation frequency. The two eigenmodes avoid being degenerate at the rotation frequency where the eigenfrequencies could be expected to cross. Instead, at that rotation frequency they go through a stage of what appears to be superpositions of the two basic eigenmodes, and the eigenfrequencies stay separated.

As expected, the eigenfrequencies are higher if a plasma rotation exists in the same direction as the mode rotation, and lower if the directions are opposite. Eigenmodes with more central localization (where the rotation is fast) tend to respond more to a change in the overall rotation frequency. An approximation to the eigenfrequency shift due to rotation can be obtained by assuming that the plasma rotates as a rigid body with a rotation frequency computed from a spatial average of the real rotation profile weighted with the eigenmode amplitude. It is, however, non-trivial to more accurately determine the magnitude of the frequency shift due to rotation without performing the full eigenmode calculation. 
With the tools used in this work, extension of the computational domain to the wall could only be made by greatly simplifying the eigenmode equation. The low effective rotation frequency discussed above could therefore not be observed in the calculations with the boundary at the wall. The extension of the domain to the wall did, however, lower all eigenfrequencies by $\sim 10-20 \mathrm{kHz}$, irrespective of the rotation frequency. This is of the same order as the frequency shifts seen from including the Hall term and the $\boldsymbol{J}_{0}$ terms in CAE3B.

\section{Acknowledgments}

The authors thank David Gates, Neal Crocker and Per Helander for fruitful discussions.

\section{Appendix A.}

The two first lines of the eigenmode equation are given (without the factors $K$ and $K^{2}$ ) in the appendix of [13]. For brevity, they are in the following expressions therefore only marked as "known terms". The component of the eigenmode equation along $\nabla r$ is $\omega^{2} g^{r r} b_{r}=$ known terms +

$-i K^{3} G \frac{\Omega}{\omega}\left(\boldsymbol{B}_{0} \cdot \nabla\right)$

$\left(\frac{g^{r r} \nu}{B_{0}^{2}}\left[-I \tilde{p}^{\prime} g^{r r} b_{r}-\psi^{\prime}\left(-B_{0}^{2} \boldsymbol{\Lambda} \cdot \nabla b_{\|}+B_{0}^{2} g^{r r} \boldsymbol{B}_{0} \cdot \nabla b_{\wedge}+B_{0}^{2} g^{r r} \alpha b_{r}+b_{\wedge} \boldsymbol{B}_{0} \cdot \nabla g^{r r}\right)\right]\right)+$

$+i \omega K \Omega g^{r r} \nu\left(b_{\wedge} \psi^{\prime} g^{r r}-I b_{\|}\right)$,

where $g^{r r} \equiv|\nabla r|^{2}$ and where $\tilde{\boldsymbol{J}}_{0} \equiv \alpha \boldsymbol{B}_{0}+\tilde{p}^{\prime} B_{0}^{-2} \boldsymbol{\Lambda}$ defines $\alpha$ and $\tilde{p}^{\prime}$. The component along $\boldsymbol{\Lambda}$ is

$$
\begin{aligned}
& \omega^{2} B_{0}^{2} g^{r r} b_{\wedge}=\text { known terms }+ \\
& -g^{r r} K^{2} G \psi^{\prime}\left[\Omega \boldsymbol{B}_{0} \cdot \nabla \nu+\left(\Omega^{\prime}+\nu \Omega\right) \boldsymbol{B}_{0} \cdot \nabla\right] \\
& \left(-B_{0}^{2} \nabla r \cdot \nabla b_{\|}+2 b_{\|} \tilde{p}^{\prime} g^{r r}+g^{r r}\left(\boldsymbol{B}_{0} \cdot \nabla\right) b_{r}-2 \boldsymbol{\Lambda} \cdot \boldsymbol{D} b_{\wedge}\right)+ \\
& +K^{2} \Omega G \tilde{p}^{\prime} g^{r r}\left[\boldsymbol{B}_{0} \cdot \nabla\left(g^{r r} \mu\right)-I B_{0}^{-2} \nu\left(B_{0}^{2} \alpha g^{r r}-2 \boldsymbol{D} \cdot \boldsymbol{\Lambda}\right)+g^{r r} \mu \boldsymbol{B}_{0} \cdot \nabla\right] b_{r}+ \\
& -K^{2} \Omega G \psi^{\prime} \nu\left(B_{0}^{2} \alpha g^{r r}-2 \boldsymbol{D} \cdot \boldsymbol{\Lambda}\right)\left(-\boldsymbol{\Lambda} \cdot \nabla b_{\|}+g^{r r} \boldsymbol{B}_{0} \cdot \nabla b_{\wedge}+g^{r r} \alpha b_{r}+b_{\wedge} B_{0}^{-2} \boldsymbol{B}_{0} \cdot \nabla g^{r r}\right)+ \\
& -i \omega K g^{r r}\left[\psi^{\prime} g^{r r}\left(\Omega^{\prime}+\nu \Omega\right) b_{r}+\Omega \mu B_{0}^{2} b_{\|}\right],
\end{aligned}
$$

where $\boldsymbol{D} \equiv\left(\boldsymbol{B}_{0} \cdot \nabla\right) \nabla r$. Finally, the component along $\boldsymbol{B}_{0}$ is

$\omega^{2} B_{0}^{2} b_{\|}=$known terms +

$$
\begin{aligned}
& G K^{3}\left[\left(i \psi^{\prime} \frac{\Omega}{\omega} \boldsymbol{\Lambda} \cdot \nabla \nu-\frac{n \Omega^{\prime}}{\omega} B_{0}^{2}\right)+i \psi^{\prime} \frac{\Omega^{\prime}+\nu \Omega}{\omega} \boldsymbol{\Lambda} \cdot \nabla\right] \\
& \left(-B_{0}^{2} \nabla r \cdot \nabla b_{\|}+2 b_{\|} \tilde{p}^{\prime} g^{r r}+g^{r r}\left(\boldsymbol{B}_{0} \cdot \nabla\right) b_{r}-2 \boldsymbol{\Lambda} \cdot \boldsymbol{D} b_{\wedge}\right)+ \\
& -i K^{3} G \frac{\Omega}{\omega} \tilde{p}^{\prime}\left[\boldsymbol{\Lambda} \cdot \nabla\left(g^{r r} \mu\right)+g^{r r} \mu \boldsymbol{\Lambda} \cdot \nabla\right] b_{r}+ \\
& -i K^{3}\left[\left(\boldsymbol{H} \cdot \boldsymbol{B}_{0} \frac{\Omega}{\omega}+g^{r r} K \frac{\Omega^{\prime}}{\omega}\right) G \nu B_{0}^{-2} I \tilde{p}^{\prime} g^{r r}+B_{0}^{2} \frac{\Omega}{\omega} \nabla r \cdot \nabla\left(G \nu B_{0}^{-2} I \tilde{p}^{\prime} g^{r r}\right)+\right.
\end{aligned}
$$




$$
\begin{aligned}
& \left.\frac{\Omega}{\omega} G \nu I \tilde{p}^{\prime} g^{r r} \nabla r \cdot \nabla\right] b_{r}+ \\
& -i K^{3}\left[\left(\boldsymbol{H} \cdot \boldsymbol{B}_{0} \frac{\Omega}{\omega}+g^{r r} K \frac{\Omega^{\prime}}{\omega}\right) G \nu \psi^{\prime}+B_{0}^{2} \frac{\Omega}{\omega} \nabla r \cdot \nabla\left(G \nu \psi^{\prime}\right)+\frac{\Omega}{\omega} G \nu \psi^{\prime} B_{0}^{2} \nabla r \cdot \nabla\right] \\
& \left(-\boldsymbol{\Lambda} \cdot \nabla b_{\|}+g^{r r} \boldsymbol{B}_{0} \cdot \nabla b_{\wedge}+g^{r r} \alpha b_{r}+b_{\wedge} B_{0}^{-2} \boldsymbol{B}_{0} \cdot \nabla g^{r r}\right)+ \\
& +i \omega K g^{r r}\left[I b_{r}\left(\Omega^{\prime}+\nu \Omega\right)+\Omega B_{0}^{2} \mu b_{\wedge}\right],
\end{aligned}
$$

where $\boldsymbol{H} \equiv \nabla \times \boldsymbol{\Lambda}=-2 \boldsymbol{D}+\alpha \boldsymbol{\Lambda}+\left(\nabla^{2} r-\tilde{p}^{\prime} g^{r r} / B_{0}^{2}\right) \boldsymbol{B}_{0}$.

[1] Coppi B, Cowley S, Kulsrud R, Detragiache P and Pegoraro F 1986 Phys. Fluids 29 4060-4072

[2] Fülöp T, Kolesnichenko Y I, Lisak M and Anderson D 1997 Nucl. Fusion 37 1281-1293

[3] Cottrell G A and Dendy R O 1988 Phys. Rev. Lett. 60 33-36

[4] Seki M, Saigusa M, Nemoto M, Kusama K, Tobita T, Kuriyama M and Uchara K 1989 Phys. Rev. Lett. 62 1989-1992

[5] Cauffman S and Majeski R 1995 Review of Scientific Instruments 66 817-819

[6] McClements K G, Hunt C, Dendy R O and Cottrell G A 1999 Phys. Rev. Lett. 82 2099-2102

[7] McClements K G, Gryaznevich M P, Sharapov S E, Akers R J, Appel L C, Counsell G F, Roach C M and Majeski R 1999 Plasma Phys. Control. Fusion 41 661-678

[8] Fredrickson E D, Gorelenkov N, Cheng C Z, Bell R, Darrow D, Johnson D, Kaye S, LeBlanc B and Menard J 2001 Phys. Rev. Lett. 87145001

[9] Gryaznevich M, Sharapov S, Lilley M, Pinches S, Field A, Howell D, Keeling D, Martin R, Meyer H, Smith H, Vann R, Denner P, Verwichte E and the MAST Team 2008 Nucl. Fusion 48084003

[10] Appel L C, Fülöp T, Hole M J, Smith H M, Pinches S D, Vann R G L and The MAST Team 2008 Plasma Phys. Control. Fusion 50115011

[11] Heidbrink W, Fredrickson E, Gorelenkov N, Rhodes T and Zeeland M V 2006 Nucl. Fusion 46 324-334

[12] Mahajan S M and Ross D W 1983 Phys. Fluids 26 2561-2564

[13] Smith H and Verwichte E 2009 Plasma Phys. Control. Fusion 51075001

[14] Gorelenkov N N, Cheng C Z, Fredrickson E D, Belova E, Gates D, Kaye S, Kramer G J, Nazikian R and White R 2002 Nucl. Fusion 42 977-985

[15] Smith H, Fülöp T, Lisak M and Anderson D 2003 Phys. Plasmas 10 1437-1442

[16] Gorelenkov N, Fredrickson E, Heidbrink W, Crocker N, Kubota S and Peebles W 2006 Nuclear Fusion 46 S933-S941

[17] Sharapov S E, Lilley M K, Akers R, Ayed N B, Cecconello M, Cook J W C, Cunningham G, Verwichte E and MAST Team 2014 Phys. Plasmas 21082501

[18] Belova E V, Gorelenkov N N, Fredrickson E D, Berk H L, Kramer G J and Medley S S 2013 Proc. of the 24th Fusion Energy Conference 2012, San Diego (Vienna: IAEA) pp TH/P6-16

[19] Fredrickson E D, Gorelenkov N N, Podesta M, Bortolon A, Crocker N A, Gerhardt S P, Bell R E, Diallo A, LeBlanc B, Levinton F M and Yuh H 2013 Phys. Plasmas 20042112

[20] Fredrickson E D, Gorelenkov N N, Belova E, Crocker N A, Kubota S, Kramer G J, LeBlanc B, Bell R E, Podesta M, Yuh H and Levinton F 2012 Nucl. Fusion 52043001 